\title{
Physiological tremor enhanced by manoeuvres affecting the segmental stretch reflex
}

\author{
ROBERT R YOUNG AND KARL-ERIK HAGBARTH* \\ From the Clinical Neurophysiology Laboratory, Department of Neurolog y, Harvard Medical School, \\ and Massachusetts General Hospital
}

SUMMARY In view of recent evidence that physiological tremor can be enhanced by positive feedback via the segmental stretch reflex, several manoeuvres and procedures were employed to enhance the finger and hand tremor of healthy subjects-the purpose being to determine if tremorogenic effects, at least in part, are due to increased efficacy of the stretch reflex servo. Mechanical events during tremor (and during voluntary or electrically induced muscle twitches) were recorde. 1 together with EMG activity from wrist and finger flexor muscles and discharges from primary spindle endings in these muscles. Physiological tremor can be enhanced not only by manoeuvres which increase the gain of segmental stretch reflexes (Jendrassik manoeuvre) but also by manoeuvres which increase the contrast in spindle firing during stretch versus shortening phases of tremor, thus enhancing reflex modulation. Effects of the latter type can be achieved by procedures which alter mechanical twitch properties of extrafusal fibres (isoproterenol infusions and fatigue) and by procedures which involve application of spindle stimuli acting preferentially during stretch phases of tremor movements (muscle vibrations). Physiological tremor, which can be temporarily enhanced by an externally applied muscle perturbation, also becomes accentuated by those small "pseudo-myoclonic" jerks which occur in all normal subjects attempting to perform slow, smooth movements.

The low-amplitude 8 to $12 \mathrm{~Hz}$ physiological action tremor present in the outstretched hands of all healthy subjects is enhanced (ie its amplitude is increased) when the contracting muscles are fatigued after a prolonged contraction and when the subject becomes frightened, excited or anxious (for historical review see Brumlik and Yap). ${ }^{1}$ Similar tremorogenic effects are produced by excessive circulating levels of thyroid hormone, epinephrine ${ }^{2}$ or the classical beta-adrenergic agonist isoproterenol. This latter agent has been shown to produce tremor by stimulation of beta-adrenergic receptors in the forearm $^{3-4}$ which are presumed to be within the contracting muscle itself. Pre-existing physiological tremor is also enhanced during muscle vibration ${ }^{5}$ and during execution of slow voluntary movements. ${ }^{6}$

Recently Hagbarth aad Young ${ }^{7}$ reported that even though the segmental stretch reflex appears not to be a driving force behind low amplitude physiological tremor, ${ }^{8}$ human primary muscle

Address for reprint requests: Dr $\mathbf{R} \mathbf{R}$ Young, Department of Neurology, Massachusetts General Hospital, Boston, Mass. 02114

Accepted 26 September 1979 spindle endings are sufficiently sensitive that they continuously monitor changes in muscle length associated with that tremor. Hagbarth and Young? also found that the segmental stretch reflex does play an important role in the maintenance of enhanced physiological tremor during fatigue and for a second or two following a sudden brief twitch or perturbation of muscle length.

In the present study, several manoeuvres have been employed to accentuate the finger and hand tremor of healthy subjects - the main purpose being to determine if the increase in tremor can be explained in terms of increased efficacy of the stretch reflex servo.

\section{Materials and methods}

As in the previous study, ${ }^{7}$ data were obtained in eight experiments in two healthy subjects (whose informed consent was obtained), from 19 recordings of neural activity identified as being of muscle spindle origin. Enphasis was placed upon multi-unit recordings from fascicles of the left median nerve innervating the wrist and finger flexor muscles but 
single unit recordings, proven to be from primary spindle endings in these muscles, were also made. Techniques of recording, amplifying and displaying neural as well as EMG activity and joint movement, isometric torque or acceleration were the same as described earlier. Unit frequency was displayed with an instantaneous frequency meter. ${ }^{9}$ As noted previously, special care was taken to ensure the EMG was recorded from the same region of the same muscle from which the spindle afferent activity came.

\section{EXPERIMENTAL SITUATION}

As in the previous study, wrist or finger tremor in the left hand was recorded with the subject lying supine, his left arm resting in an abducted-supinated position on a support reaching down to the wrist. The unsupported left hand was held outstretched against gravity with or without a weight of $40 \mathrm{~g}$ attached to the fingers. On a few occasions, tremor was recorded isometrically with the subject pressing his fingers against a force transducer.

\section{MANOEUVRES AND DRUG INJECTIONS}

Effects of exercise or fatigue were studied by adding extra weights $(200,400$ or $2500 \mathrm{~g})$ on the outstretched fingers or hand for 1 to 20 minutes. Beta adrenergic stimulation was achieved by intravenous infusion of isoproterenol, diluted in saline and infused by a Harvard pump ( 4 to $5 \mu \mathrm{g} / \mathrm{min}$ ) through an indwelling venous cannula in the right arm. Vibratory stimulation was delivered to muscle belly or tendon by manual application of a modified pneumatic drill (Dayton model 2Z491A) to which an eccentric weight was attached. Vibration amplitude was 1 to $1.5 \mathrm{~mm}$ and the frequency of vibration was continuously adjustable. Jendrassik manoeuvres consisted of the subject making a tight fist with the contralateral right hand, care being taken that he did not at the same time increase the force of contraction in the left wrist and finger flexors. Slow isotonic voluntary flexion and extension movements of wrist or one joint of one finger were studied using the goniometer described before. The subject watched a low level DC signal on an oscilloscope to guide him in the production of the desired joint movement; for analysis, these tape recorded signals were also amplified further by the TECA Instagraph (FOR 4) using an AC circuit (time constant $0.33 \mathrm{sec}$ ) to permit analysis of sudden small deviations from the baseline. Electrical twitches were produced by $0.5 \mathrm{~ms}$ square wave pulses delivered from a Grass stimulator through the EMG electrodes.

\section{Results}

As outlined in our previous paper, ${ }^{7}$ recordings of multi-unit neural activity were made to obtain an "integrated" view of muscle spindle afferent discharge during these tremors. Both multi-unit recordings and single unit recordings were demonstrated respectively to reflect predominantly or exclusively the discharge of muscle spindle afferent endings, using classical criteria for identification.

TREMOR ENHANCED BY EXERCISE

As depicted in fig 1 , the amplitude of physiological tremor was considerably increased by prolonged tonic contraction of muscles (compare $1 \mathrm{~A}$ and $1 \mathrm{~B}$ ). This enhanced physiological tremor differed from that seen at the beginning of contraction by virtue of both increased "afferent tremor" in response to the stretch phases of tremor cycles and obvious grouping of EMG potentials. The beginning and ending of each EMG burst followed approximately $20 \mathrm{~ms}$ after the homologous phase of the grouping of afferent discharges in the neurogram (fig 1C).

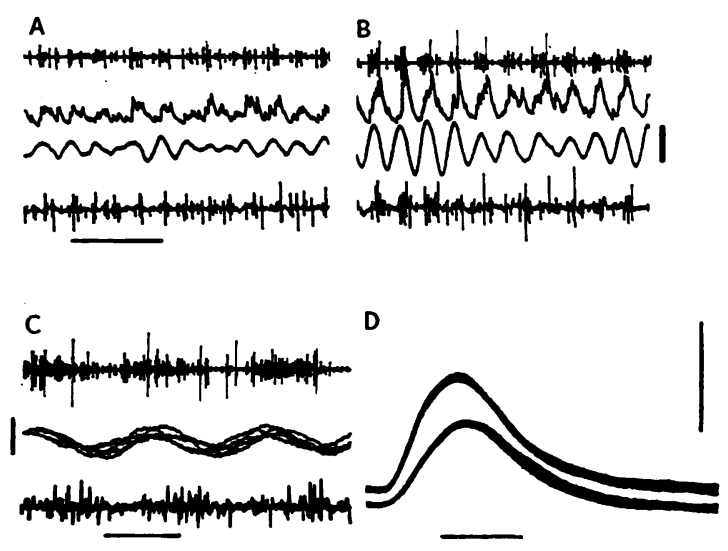

Fig 1 Effect of "fatigue" on physiological tremor. A and $B$ show multi-unit neural activity, integrated neural activity, accelerometer output and EMG (top to bottom respectively) from a wrist flexor muscle. $A$ is a few seconds and $B 20$ minutes after lifting the unloaded hand and holding it outstretched against gravity. Calibrations are $0.2 \mathrm{~g}$ and $500 \mathrm{~ms}$. Note an increase in $B$ in both tremor amplitude and afferent tremor during stretch phases. $C$ is five superimposed recordings of multi-unit neural activity, acceleration and EMG from the same muscle as $A$ and $B$ during "fatigue tremor". Calibrations are $0.2 \mathrm{~g}$ and $100 \mathrm{~ms}$. Note relationships between neural and EMG bursts. D shows five superimposed recordings of isometric torque produced by $0.5 \mathrm{~Hz}$ electrically induced twitches of a finger flexor muscle before (below) and after (above) $a$ 10 min moderate voluntary isometric contraction of that muscle. Calibrations $=0.5 \mathrm{~N}-\mathrm{m}$ and $100 \mathrm{~ms}$. Note posttetanic potentiation of twitch amplitude and shortening of contraction and half-relaxation times. 
Mechanical properties of the extrafusal fibres were also altered by their sustained contraction. During supramaximal electrically induced twitches of these fibres, the time from onset to peak tension (contraction time) was reduced by approximately $10 \%$, the half-relaxation time shortened by almost $15 \%$ and the peak force increased by $40 \%$ in comparison with similar twitches in the same fibres when they were not "fatigued" (fig 1D). No recordings were made of spindle discharges during the falling phase of these enhanced twitch contractions, but there is little reason to doubt that these larger twitches with steeper falling phases were accompanied by stronger and more synchronised spindle discharges than were the control twitches (cf. fig 2B and $\mathrm{C}$ ).

\section{TREMOR ENHANCED BY BETA ADRENERGIC}

STIMULATION

The amplitude of physiological tremor and the magnitude of the associated afferent "tremor" were also both increased by circulating isoproterenol, the classical beta-adrenergic agonist (fig 2A and D). Afferent signals accompanying a series of quick voluntary twitches of approximately the same amplitude and time course were studied before and during isoproterenol administration (fig $2 \mathrm{~B}$ and $\mathrm{E}$ ). In agreement with earlier observations, ${ }^{11}$ two bursts of spindle discharges were seen during each voluntary twitch; an initial burst during the contraction phase and a second one during the falling phase of the twitch. Following isoproterenol administration no obvious changes were seen in the initial bursts of afferent activity generated by alpha-gamma coactivation, but there was a tendency for the second (stretch) discharges to be more synchronised. There were also changes in the mechanical aspects of the voluntary "twitch" with beta-adrenergic stimulation, particularly a slight shortening of the relaxation phase.

Electrically induced twitches were also produced to study alterations of mechanical properties and changes in afferent input produced by isoproterenol. As seen in fig $2 \mathrm{C}$ and $\mathrm{F}$, such stimulation was associated with a decrease in both contraction and halfrelaxation times. Particularly impressive was a marked shift in timing so that the bulk of the afferent volleys arose at earlier portions of the stretch phases.

In several other experiments, studies were made of the multi-unit responses of muscle afferents to standard quick muscle stretches in the form of tendon taps or passive phasic muscle stretch. During isoproterenol administration, we were unable to detect any alterations in afferent discharge from muscle in response to these stimuli.

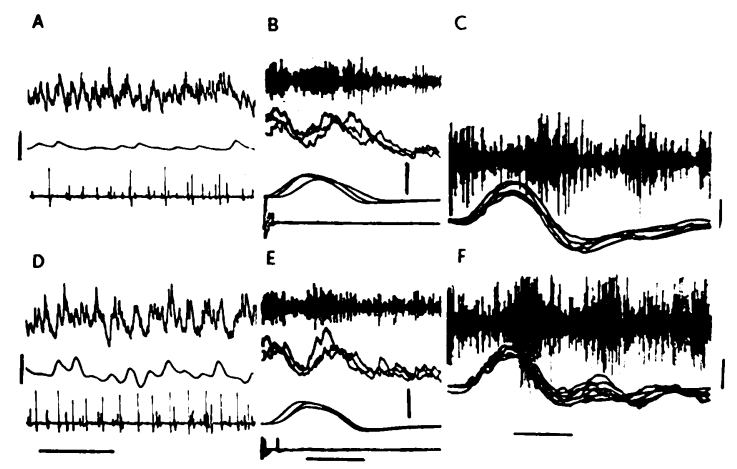

Fig 2 Effects of isoproterenol. In each column upper recordings are before and lower recordings during intravenous isoproterenol infusion. In column $A$, physiological tremor and its enhancement with isoproterenol are recorded by a goniometer (middle line in each) with the index finger outstretched. The bottom line is EMG and the top line integrated multi-unit neural activity (time constant $0.02 \mathrm{~s}$ ) from the flexor muscle of the distal phalanx. Calibrations are 0.5 degree and $500 \mathrm{~ms}$. In $B$, three single voluntary isotonic twitches from the same muscle are superimposed. The three lower lines are the same as in $A$, the uppermost line in $B$ is original multi-unit neural activity. Calibrations are 2 degrees and $100 \mathrm{~ms}$. Note separate contraction and stretch discharges from spindles. In $C$, superimposed recordings are made of electrically induced twitches in a wrist flexor muscle. The bottom line is the goniometer signal from the wrist and the upper line multi-unit neural activity from the same muscle. Calibrations are 1 degree and $100 \mathrm{~ms}$. Note pause in spindle discharge during shortening phases of the twitch with bursts of activity during stretch phases. Note also tremor on the lower mechanogram. For further details see text.

VIBRATION ENHANCED TREMOR

Vibrations at a number of different frequencies applied to flexor muscles or their tendons, increased the amplitude (but did not alter the frequency) of physiological tremor and also the amplitude of naturally occurring unwanted jerky irregularities of movement (see below) (fig 3A and B). Similar effects of vibration were also demonstrated for other muscles such as knee extensors and flexors. As the tremulous movements increased in amplitude, so did the striking mirror-image representation of them in the afferent neurogram.

To investigate mechanisms responsible for this enhancement of tremor by vibration, single unit recordings from spindle afferents were made which confirmed earlier observations reported by Burke et al. ${ }^{12}$ In fig $3 \mathrm{C}$ and $\mathrm{D}$, the spindle ending was stiunlated by a $90 \mathrm{~Hz}$ vibrator applied to the muscle tendon. With the finger relaxed (fig $3 \mathrm{C}$ ), the 
A

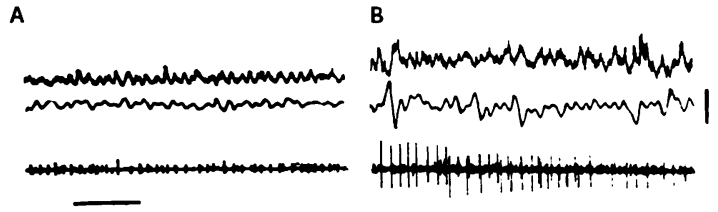

C

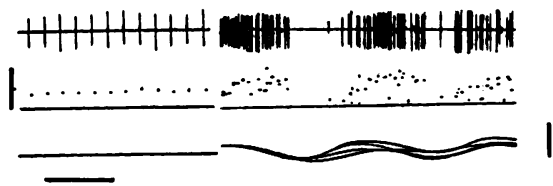

Fig 3 Effect of vibration on physiological tremor. A and $B$ show integrated multi-unit neural activity, goniometer recording and EMG (from above down) from a wrist flexor muscle before $(A)$ and during $(B)$ sustained vibration $(90 \mathrm{~Hz})$ of the appropriate tendon during a slow isotonic flexor movement. Calibrations $=0.3$ degrees and $1 \mathrm{~s}$. Note enhancement of both tremor and less rhythmic irregularities (cf fig 5) during vibration. A remarkable "mirror-image" relationship exists between neural and mechanical activity, neural bursts occurring during each stretch phase. $C$ and $D$ are single unit recordings from a primary spindle ending in the flexor digitorum profundus muscle of the index finger. In $C$ the muscle is relaxed and during $D, 5$ per $s$ passive low amplitude flexion-extension movements are superimposed. The instantaneous frequency plot of spindle discharge is in the middle and the goniometer (at low gain) on the bottom. Calibrations are $0-100 \mathrm{~Hz}, 5$ degrees and $100 \mathrm{~ms} . C$ is one and $D$ four superimposed recordings during sustained vibration of the appropriate tendon. See text for details.

spindle ending was activated by every other vibratory wave (at $45 \mathrm{~Hz}$ ). When, under the same circumstances, the distal interphalangeal joint was passively flexed and extended a few degrees in an oscillatory tremor-like manner (fig 3D), the same vibration activated the primary ending in a 1 to 1 fashion (at $90 \mathrm{~Hz}$ ) during the stretch phases of each "tremor cycle" whereas it failed to activate it at all during the shortening phases.

\section{TREMOR ENHANCED BY THE JENDRASSIK}

MANOEUVRE

Strong voluntary contraction of muscles in the right arm increased the amplitude of action tremor in the wrist and finger flexor muscles of the left arm even though there was no obvious increase in the number of motor units activated in the latter muscles. Fig 4 shows an isometrically recorded wrist tremor being enhanced by a Jendrassik manoeuvre without any net increase in the force produced by the wrist flexors. Tremor amplitude was not increased to the

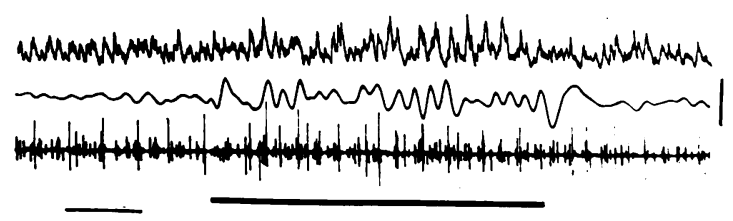

Fig 4 Effects of a Jendrassik manoeuvre (during the time denoted by the thick black line) on action tremor recorded by a strain gauge (middle line-calibration = $2 N-m$ ) during sustained isometric voluntary contraction of wrist flexors. Integrated multi-unit neural activity (time constant $0.02 \mathrm{~s}$ ) and EMG from the appropriate muscle are displayed on the upper and lower lines respectively. Time calibration $500 \mathrm{~ms}$. Note the increase tremor and "tremulous" neural activity during the Jendrassik manoeuvre despite no obvious increase in baseline torque or number of motor units active in the EMG.

same extent merely by contracting the left wrist flexors more forceably. As seen in fig 4, the "afferent tremor" in the neurogram reflects the increased fluctuations of torque, bursts of neural activity occurring during the relaxation phase of each tremulous contraction.

\section{INCREASE IN TREMOR AND INVOLUNTARY JERKS DURING VOLUNTARY MOVEMENTS}

Fig 5A demonstrates the enhancement of tremor that regularly occurred when, instead of maintaining a body part in one posture, the subject made slow flexion and extension movements, striving to keep the speed of movement constant. High-gain goniometer recordings revealed that in addition to the enhanced tremor which is particularly prominent during shortening of the muscle, there were other involuntary, randomly occurring, small-amplitude irregularities in the speed of movement. ${ }^{13}$ These sudden jerks were, with few exceptions, in the direction of movement: excessive flexion during the flexion phase of the slow movement and excessive extension during the extension phase. Simultaneous EMG recordings showed that most of the jerks occurring during the slow flexion movements were preceded by brief periods of grouped EMG activity (fig 5B) - the resultant jeiks being brief flexion twitches in which the EMG activity was accompanied by a burst of spindle afferent discharge. Most of the jerks occurring during slow extension movements were preceded by brief periods of relative EMG silence in flexor muscles (fig 5C), the resultant jerks being brief extensions. Both tremor and the other irregularities of movement were larger when weights were added to the moving part. Superimposed recordings of these jerky movements 


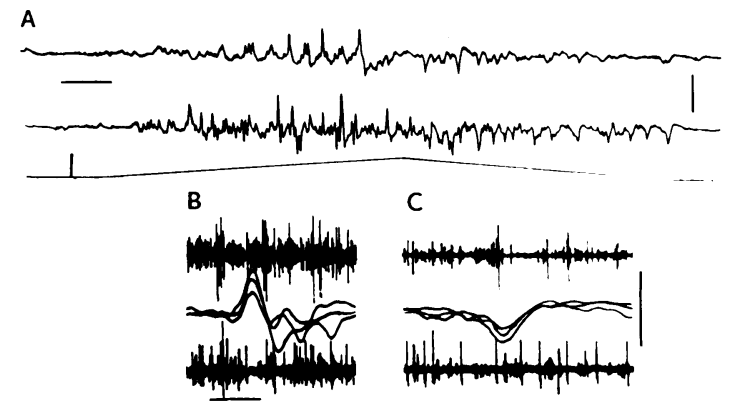

Fig 5 Upper two lines in A are AC coupled high gain goniometer recordings from the distal interphalangeal joint of the index finger during a slow voluntary 30 degree flexion and extension movement, third line shows the extent and time course of the movement. Calibrations are $2 \mathrm{~s}, 0.5$ degree for the top two lines and 180 degrees to 150 degrees on bottom line. Top line is with index finger unloaded; 2nd line is with palmar aspect of distal phalanx supporting a weight of $40 \mathrm{~g}$. Note increases in both tremor and less rhythmic irregularities in speed during slow "smooth" voluntary movement which are more marked when the finger supports a weight. Irregularities are predominantly in direction of movement. $B$ and $C$ are superimposed recordings of irregularities in direction of flexion $(B)$ and extension $(C)$ together, simultaneous neural recordings (upper lines) and EMG (lower lines) from the flexor of distal joint of index finger. Middle lines are goniometer recordings arranged as in $\mathrm{A}$ except the calibrations are $200 \mathrm{~ms}$ and 0.5 degree. Note neural discharges during the beginning of the stretch phases and the excessive recruitment $(B)$ or derecruitment $(C)$ just before the movements.

(fig 5B during flexion and 5C during extension) showed bursts of afferent discharge occurring during the stretch phases. These were followed in approximately $20 \mathrm{~ms}$ by resumption of EMG discharges which were relatively scarce just preceding each stretch phase. Similar irregularities have been recorded at many joints, proximal and distal and in upper and lower extremities.

\section{Discussion}

PHYSIOLOGICAL TREMOR ENHANCED BY TONIC CONTRACTION

The amplitude of physiological tremor increases considerably (and for a short time following) the course of a tonic muscle contraction ${ }^{14}$ even if the latter is of rather modest intensity (such as supporting the weight of one's hand against gravity) and unaccompanied by any sense of effort or fatigue. An increase in amplitude is obvious within a few minutes of beginning a contraction, long before the subject feels fatigued. For these reasons, the term "fatigue tremor", often used to describe this type of enhanced physiological tremor, seems not entirely appropriate.

Whereas grouping of EMG discharges is not obvious at the beginning, it does become apparent as the tonic contraction progresses. The timing of these EMG bursts, each of which follows a burst of primary spindle afferent input from the tremulous muscles, suggests that the segmental stretch reflex is involved in sustaining the enhanced tremor whereas it is not obviously involved in the generation of the the initial low-level physiological tremor. Present findings thus agree with previous deductions about the segmental stretch reflex as a modulator of the motor outflow during enhanced physiological tremor. ${ }^{7}$ The act of voluntarily holding the supinated hand outstretched against gravity involves a sustained descending excitatory drive on both alpha and gamma motoneurones supplying the flexor muscles ${ }^{15-17}$ With the oscillatory tendencies inherent in the mechanical part of the system and the high sensitivity of the fusimotor-driven spindle endings to minute oscillations, rhythmical volleys of impulses from spindles are propagated back to the alpha motoneurones tending to synchronise the motor discharges in such a way that the rhythmical outflow helps keep the oscillatory movements going.

The question remains, what fundamental change takes place as the contraction proceeds which eventually alters the afferent input and/or the gain of the stretch reflex to produce the synchronisation of EMG activity which is presumed to be responsible for the increase in tremor amplitude? The present demonstration of exercise-induced alterations of the twitch characteristics of extrafusal fibres suggests that peripheral mechanical mechanisms might be responsible for the enhancement of the "afferenttremor" and EMG synchrony.

Tetanisation of a muscle nerve may be followed by a long lasting increase in tension produced by a twitch in that muscle ${ }^{18}$ and twitch contraction force may also increase as much as $50 \%$ when muscle nerve stimuli are repeated at 2 to 3 per s for several minutes..$^{19-21}$ This "stair-case phenomenon" is also associated with a significant decrease in contraction time and in half-relaxation time. In their studies of mechanical responses of thenar muscles to single supramaximal stimuli following 1 minute of maximal voluntary contraction. Slomic et $a l^{21}$ report a modest "postactivation potentiation" of twitch force maximal 2 to 4 minutes after the effort. During the first 30 seconds after cessation of voluntary contraction, they recorded a decrease in peak force. Ischaemic or other intramuscular causes of "exhaustion" may have complicated the experimental results, making comparison difficult 
to our studies of the effects of 10 minutes of moderate voluntary isometric contraction in finger flexor muscles (fig 1D).

Our experiments demonstrate postactivation potentiation of twitch amplitude as well as changes in contraction and half-relaxation times similar to those seen during the "staircase phenomenon". Changes in timecourse of the mechanical response were not reported by Slomic et $a l^{21}$ and these relationships must be studied in further detail. However, an increase in force and/or a decrease in the duration of each twitch-like phase of a cycle of physiological tremor would produce a larger and slightly earlier burst of spindle primary afferent discharges and stronger EMG synchronisation.

Other mechanisms than those referred to above may also contribute to the tremor enhancement during sustained contraction. For instance, one cannot exclude potentiation of the stretch reflex itself as demonstrated by Hagbarth ${ }^{22}$ in his study of posttetanic potentiation of myotatic reflexes in man. This suggestion implies that such an increase in central excitability may also occur following the increased afferent input associated with alphagamma coactivation during exercise. ${ }^{23}$

Where exactly does the boundary lie between physiological tremor and exercise enhanced physiological tremor? The former in which the segmental stretch reflex appears not to be active appears to merge gradually as time passes into the latter in which the stretch reflex plays a large role. Difficulty distinguishing between these two and the fact that the latter inevitably develops as an experiment proceeds may account for the considerable controversy in the literature over whether or not "physiological tremor" (some combination of the two) involves synchronisation between individual motor units ${ }^{8}$ and requires the participation of muscle spindles and the stretch reflex servo-loop. ${ }^{24}$

\section{PHYSIOLOGICAL TREMOR ENHANCED BY BETA-}

\section{ANDRENERGIC STIMULATION}

Stimulation of peripheral beta (probably beta-2) adrenergic receptors within a limb produces an action tremor. $^{34}$ Stimulation of beta-adrenergic receptors on extrafusal muscle fibres also changes the mechanical properties of muscles ${ }^{25-27}$ prolonging the "active state" of fast-twitch fibres and shortening the "active state" of slow-twitch ones. The half-relaxation time of an electrically induced twitch of cat soleus and human calf muscles is reduced by isoproterenol. In addition, both the tension and "degree of fusion" of incomplete tetanic contractions are also reduced. These latter mechanical changes might render a tonic contraction tremulous but would not produce bursts in the EMG unless afferent input at the segmental level was also altered.

Our observations confirm both the enhancement of tremor amplitude and decrease in contraction and half-relaxation time (especially noticeable during an electrically induced twitch but probably also present with voluntary twitches-fig 2) following isoproterenol infusion. We were also able to document increased grouping of spindle afferent discharges during the relaxation phases of individual twitches or tremor cycles. Thus the intramuscular changes produced by isoproterenol are similar to those described above with exercise and similar arguments apply for increased afferent efficacy at the segmental stretch reflex level. It remains uncertain whether, in addition to these changes, isoproterenol may also produce some change in the mechanical properties or sensitivity of the muscle spindles themselves. We could not find a primary alteration in spindle sensitivity (in terms of afferent response to phasic stretches of muscle) or in the presence of early contraction discharges from the spindles during the voluntary phasic twitches (alphagamma coactivation). Still it cannot be definitely excluded that sensitisation of spindle endings contributed to the marked alterations in timing of afferent stretch responses during the falling phase of the twitches (fig $2 \mathrm{C}$ and F). At any rate, the observed grouping of motoneurone discharges during adrenergically enhanced tremor would be expected to result from altered input to the segmental stretch reflex caused by changes in mechanical properties of extrafusal muscle fibres. This evidence suggests that adrenergically enhanced tremor is closely related to physiological tremor enhanced by exercise and is not yet another, clearly different type of tremor.

\section{PHYSIOLOGICAL TREMOR ENHANCED BY VIBRATION} Vibration is a most effective method for stimulating primary muscle spindle endings. ${ }^{12} 2829$ Homma et $a l^{30}$ have shown, during the tonic vibratory reflex, the firing intervals of motoneurones to coincide in a statistically significant way with one or other integer multiple of the period ( 1 /frequency) of vibration being applied. On the other hand, vibration of muscle at various frequencies has been reported to enhance different types of rhythmical involuntary movements such as clonus, ${ }^{23}{ }^{31}$ resting Parkinson tremor ${ }^{23}$ and physiological tremor. ${ }^{5}$ Our findings (cf fig 3 ) confirm the vibiatory enhancement of physiological tremor (and of other irregular jerky movements - see below) during a slow "smooth" flexion movement and document the strikingly enhanced mirror image representation in the neurogram of the mechanical 
changes, afferent bursts occurring on the stretch phase of each movement.

Burke et $a l^{12}$ demonstrated that the sensitivity of human spindle primary endings, as measured by their response to vibration, is a function of the degree of stretching or shortening of the muscle. This observation was confirmed in the present study, where it was also shown that small tremor-like passive movements are sufficient to produce a marked modulation of the spindle sensitivity to vibration (fig $3 \mathrm{C}$ and $\mathrm{D}$ ). With spindles pausing during shortening phases and being driven to firing rates of about $100 \mathrm{~Hz}$ during stretch phases, vibration produces very marked bursts of spindle discharges timed to the tremor-like movements. By analogy we assume that a similar modulation of vibration sensitivity occurs during ongoing physiological tremor and that vibration thus enhances the "afferent tremor" by increasing the contrast in spindle firing between shortening and lengthening phases. By virtue of its segmental reflex action, this enhanced "afferent-tremor" would produce an increase in EMG synchronisation properly timed to accentuate the ongoing tremulous movements. This dgrees with the suggestion of Cussons et al, ${ }^{5}$ that, if a small amplitude tremor is present, vibration would tend to accentuate it because of the "larger change in relevant spindle firing, and thus deeper reflex modulation".

Examples from hand and forearm as well as leg and other muscles demonstrate that vibration makes it more difficult for a person to sustain a smooth contraction without tremor and increased jerky irregularities. The vibration-induced fluctuations in afferent input to the cord described above may account for this difficulty as well as the significant decrease reported ${ }^{32}$ in a subject's ability to isolate single motor units during and after vibration or a maximal isometric contraction. Similarly it would explain the observations that vibration increases clonus in spastic patients ${ }^{31}$ and resting tremor in Parkinsonian patients $^{23}$ in whom a pre-existing tendency exists for involuntary oscillatory movements.

\section{PHYSIOLOGICAL TREMOR ENHANCED BY}

\section{JENDRASSIK MANOEUVRE}

Considering arguments put forward and following observations described above, it was of interest to test the effect of the Jendrassik manoeuvre which is known to cause a reinforcement of the segmental stretch reflex. As predicted, this manoeuvre caused a potentiation of physiological tremor, supporting the notion that enhanced physiological tremors are dependent on the efficacy of the stretch reflex servo. For the present argument it is irrelevant whether the reflex potentiation caused by the manoeuvre is due to a fusimotor induced change in muscle spindle sensitivity or to an increase in the central gain of the reflex. ${ }^{11}{ }^{33}$ In either case the manoeuvre would produce a similar enhancement of tremor amplitude ascribed above to increased "tremulousness" of the afferent input.

It may be argued that physiological tremor is enhanced during a Jendrassik manoeuvre merely because the subject unintentionally activates more motor units in the trembling muscles. Joyce and Rack $^{6}$ demonstrated increased tremor amplitude with increased force of voluntary contraction (up to $1 / 2$ or $3 / 4$ maximum). However, no increase in the mean active force of finger and wrist flexors was registered by the strain gauge in our studies (fig 4) and a voluntary increase in flexion did not enhance tremor to the same extent as the Jendrassik manoeuvre.

\section{A POSSIBLE ROLE FOR THE SEGMENTAL STRETCH REFLEX IN NORMAL MOVEMENT}

During slow, isotonic and apparently smooth voluntary contractions associated with shortening or lengthening of a muscle, two different types of unintended movement are seen in normal subjects if high gain goniometers are used (fig $5 \mathrm{~A}$ ): tremulous movements at 8 to $12 \mathrm{~Hz}$ are mixed with irregular, more randomly occurring jerks. The tremulous movements represent an enhancement of pre-existing physiological tremor (as described by Joyce and Rack $^{6}$ ) especially during shortening contractions, for which several possible explanations are avalable: (1) recruitment of more motor units, (2) increased afferent input due to increased alphagamma coactivation ${ }^{15}{ }^{34}$ which would be modulated by ongoing tremulous contractions as noted above, (3) increased central gain in the stretch reflex during increasing contraction of the prime mover ${ }^{16}$ and/or related to Jendrassik-like effects of contractions in synergistic or distant muscles, or (4) a temporary "after-tremor" following the irregular jerks similar to the damped tremulous oscillations following any mechanical perturbations superimposed on contracting muscles in freely moving limbs ${ }^{35}$ (for example, see the oscillations following the larger movements in fig 5B).

Irregular jerky alterations of the speed of movement (fig 5) were originally described by Vallbo ${ }^{13}$ and further studied by Burke et al. ${ }^{17}$ Superimposed EMG recordings, revealing flexor EMG bursts preceding the flexion jerks and short periods of relative flexor EMG silence preceding the extension jerks, suggest some inability to avoid irregularities in the output from a motoneurone pool in which gradual recruitment or derecruitment is in progress. 
EMG bursts preceding flexion jerks were accompanied by afferent spindle discharges as though these sudden involuntary enhancements in motor output involve not only the skeletomotor but also the fusimotor neurones. It is of interest to compare these normal involuntary small jerks, preceded by bursts or pauses in the EMG activity, with more prominent "positive" or "negative" myoclonic jerks observed in different pathological states. ${ }^{36}$ Pathological myoclonic jerks might possibly be regarded merely as an accentuation of errors in motor performance which are normally present during the execution of smooth voluntary movements.

Whatever the underlying mechanisms for these jerky movements may prove to be, fig $5 \mathrm{~B}$ and $\mathrm{C}$ demonstrate that they give rise to segmental stretch reflex responses as do other rapid perturbations of muscle length during movement. ${ }^{7}$ Approximately $20 \mathrm{~ms}$ after increased spindle discharge resulting from involuntary fast extension in fig 5C, an EMG discharge occurs and the movement produced compensates for the lapse of posture. Similarly, in fig 5B, the EMG becomes relatively silent about $20 \mathrm{~ms}$ after the unloading pause in afferent activity. The timing of these stretch and unloading reflexes respectively is compatible with action of segmental (rather than long-loop) stretch reflex mechanisms ${ }^{17}$ which would compensate with minimal latency for unintentional errors in recruitment or derecruitment during the production of voluntary slow smooth movements. In this way, small internal disturbances during movement ${ }^{37}$ and resultant abrupt variations in velocity of movement would be minimised as quickly as possible to produce a smoother movement, any "after-tremor" representing damped oscillations in this stretch reflex servo. ${ }^{7}$ Observations of excessively jerky movements in patients with certain neuropathies particularly affecting muscle afferent fibres ("neuropathic tremor") ${ }^{38}$ tend to support our present hypothesis.

This research was supported by the Parkinsons Disease Project of the Massachusetts General Hospital and the Swedish Medical Research Council (Project No B78-14X-02881-094A). We thank Mr Melvin Meister for his valuable technical help and the TECA Corporation for their generous assistance with equipment.

* On Sabbatical leave from the Department of Clinical Neurophysiology, University Hospital, S-75014 Uppsala, Sweden.

\section{References}

1 Brumlik J, Yap CB. Normal Tremor. Springfield: CC Thomas, 1970; 40.

2 Marsden CD, Gimlette TMD, McAllister RG,
Owen DAL, Miller TN. The effects of betaadrenergic blockade on finger tremor and Achilles reflex time in anxious and thyrotoxic patients. Acta Endocrinol 1968; 57:353-62.

3 Marsden CD, Foley TH, Owen DAL, McAllister RG. Peripheral beta-adrenergic receptors concerned with tremor. Clin Sci 1967; 33:53-65.

4 Young RR, Growdon JH, Shahani BT. Betaadrenergic mechanisms in action tremor. $N$ Eng $J$ Med 1975; 293:950-3.

5 Cussons PD, Matthews PBC, Muir RB, Watson JDG. Enhancement of human elbow tremor by muscle vibration. J Physiol 1978; 284:42-3.

6 Joyce GC, Rack PMH. The effects of load and force on tremor at the normal human elbow joint. $J$ Physiol 1974; 240:375-96.

7 Hagbarth KE, Young RR. Participation of the stretch reflex in human physiological tremor. Brain 1979; 102:509-26.

8 Freund HJ, Dietz V. The relationship between physiological and pathological tremor. In: Desmedt JE, ed. Progress in Clinical Neurophysiology vol 5, Physiological Tremor, Pathological Tremors and Clonus. Basel: Karger, 1978; 66-89.

9 Hagbarth KE, Wallin G, Löfstedt L. Muscle spindle activity in man during voluntary fast alternating movements. J Neurol Neurosurg Psychiatry 1975; 38:625-35.

10 Szumski AJ, Burg D, Struppler A, Velho F. Activity of muscle spindles during muscle twitch and clonus in normal and spastic human subjects. Electroencephalogr Clin Neurophysiol 1974; 37:589-97.

11 Hagbarth KE, Wallin G, Burke D, Löfstedt L. Effects of the Jendrassik manoeuvre on muscle spindle activity in man. $J$ Neurol Neurosurg Psychiatry 1975; 38:1143-53.

12 Burke D, Hagbarth KE, Löfstedt L, Wallin BG. The responses of human muscle spindle endings to vibration of non-contracting muscles. J Physiol 1976; 261:673-93.

13 Vallbo ÅB. Muscle spindle afferent discharge from resting and contracting muscles in normal human subjects. In: Desmedt JE, ed. New Developments in Electromyography and Clinical Neurophysiology vol 3. Basel: Karger, 1973; 251-62.

14 Stiles RN. Frequency and displacement amplitude relations for normal hand tremor. J App Physiol 1976 ; 40:44-54.

15 Vallbo ÅB. Discharge patterns in human muscle spindle afferents during isometric voluntary contractions. Acta Physiol Scand 1970; 80:552-66.

16 Burke D, Hagbarth KE, Löfstedt L. Muscle spindle responses in man to changes in load during accurate position maintenance. J Physiol 1978; 276:159-64.

17 Burke D, Hagbarth KE, Löfstedt L. Muscle spindle activity in man during shortening and lengthening contractions. J Physiol 1978; 277:131-42.

18 Botelho SY. Alterations in muscle tension without similar changes in electrical activity in patients with myasthenia gravis. $J$ Clin Invest 1955 ; 34:1403-9.

19 Desmedt JE, Hainaut K. Modifications des propriétés contractiles du muscle strié au cours de la 
stimulation électrique répétée de son nerf moteur chez l'Homme normal. $C R$ de la Academie des Sciences 1967; 264:363-6.

20 Desmedt JE, Hainaut K. Kinetics of myofilament activation in potentiated contraction: staircase phenomenon in human skeletal muscle. Nature 1968; 217:529-32.

21 Slomic A, Rosenfalck A, Buchthal F. Electrical and mechanical responses of normal and myasthenic muscle. Brain Res 1968; 10:1-78.

22 Hagbarth KE. Post-tetanic potentiation of myotatic reflexes in man. J Neurol Neurosurg Psychiatry 1962; 25:1-10.

23 Hagbarth KE, Eklund G. The effects of muscle vibration in spasticity, rigidity and cerebellar disorders. J Neurol Neurosurg Psychiatry 1968; 31: 207-13.

24 Marsden CD. The mechanisms of physiological tremor and their significance for pathological tremors. In: Desmedt JE, ed. Progress in Clinical Neurophysiology vol 5, Physiological Tremor, Pathological Tremor and Clonus. Basel: Karger, 1978; 1-16.

25 Bowman WC, Zaimis E. The effects of adrenaline, noradrenaline and isoprenaline on skeletal muscle contractions in the cat. J Physiol 1958; 144:92-107.

26 Bowman WC, Nott MW. Actions of some sympathomimetic bronchodilator and beta-adrenoceptor blocking drugs on contractions of the cat soleus muscle. Br J Pharmacol 1970; 38:37-49.

27 Marsden CD, Meadows JC. The effect of adrenaline on the contraction of human muscle. J Physiol 1970; 207:429-48.

28 Bianconi R, Van Der Meulen JP. The response to vibration of the end organs of mammaliam muscle spindles. J Neurophysiol 1963; 16: 177-90.

29 Brown MC, Engberg I, Matthews PBC. The relative sensitivity to vibration of muscle receptors of the cat. J Physiol 1967; 192: 773-800.

30 Homma S, Kanda K, Watanabe, S. Preferred spike intervals in the vibration reflex. Jpn J Physiol 1972; 2:421-32.

31 Kanda K, Homma S, Watanabe S. Vibration reflex in spastic patients. In: Desmedt JE, ed. New developments in Electromyography and Clinical Neurophysiology vol 3. Basel: Karger, 1973; 469-74.

32 Malouin F, Simard T. Vibration influence on control of single motor unit activity. Arch Phys Med Rehabil 1978; 59:144-51.

33 Burg D, Szumski AJ, Struppler A, Velho F. Assessment of fusimotor contribution to reflex reinforcement in humans. J Neurol Neurosurg Psychiatry 1974; 37:1012-21.

34 Burke D, Hagbarth KE, Wallin BG..Alpha-gamma linkage and the mechanisms of reflex reinforcement. In: Desmedt JE, ed. Progress in Clinical Neurophysiology vol 8, Spinal and supraspinal mechanism of voluntary motor control and locomotion. Basel: Karger, 1980; 170-80.

35 Lippold OCJ. Oscillations in the stretch reflex arc and the origin of the rhythmical 8-12 c/s component of physiological tremor. J Physiol 1970; 206:359-82.

36 Shahani BT, Young RR. Physiological and pharmacological aids in the differential diagnosis of tremor. J Neurol Neurosurg Psychiatry 1976; 39:772-83.

37 Evarts EV, Bizzi E. Some teleological considerations regarding myotatic reflexes. In: Desmedt JE, ed. Abstracts of Communications at the International Symposium on Human reflexes and Motor disorders. Brussels: 1976; 71-2.

38 Adams RD, Shahani BT, Young RR. Tremor in association with polyneuropathy. Trans Am Neurol Ass 1972; 97: 44-8. 